\title{
Retrieval of effective parameters for bianisotropic metamaterials with omega shaped metallic inclusions
}

\author{
Zhaofeng $\mathrm{Li}^{\mathrm{a}, *}$, Koray Aydin ${ }^{\mathrm{a}, \mathrm{b}}$, Ekmel Ozbay ${ }^{\mathrm{a}, \mathrm{b}, \mathrm{c}}$ \\ ${ }^{a}$ Nanotechnology Research Center, Bilkent University, Bilkent, 06800 Ankara, Turkey \\ ${ }^{\mathrm{b}}$ Department of Physics, Bilkent University, Bilkent, 06800 Ankara, Turkey \\ ${ }^{\mathrm{c}}$ Department of Electrical and Electronics Engineering, Bilkent University, Bilkent, 06800 Ankara, Turkey \\ Received 19 March 2011; received in revised form 17 October 2011; accepted 8 November 2011 \\ Available online 18 November 2011
}

\begin{abstract}
Bianisotropic metamaterials including $\Omega$-shaped metallic elements are investigated experimentally and theoretically. A passband is observed for a composite metamaterial (CMM) based on an $\Omega$-medium below the plasmonic frequency of the corresponding closed CMM. The effective parameters (refractive index, impedances, permittivity, permeability, and magnetoelectric coupling coefficient) are retrieved for the $\Omega$-medium and the CMM based on it. Our retrieval results show that the passband observed for the CMM is a band with positive refractive indices. Our retrieval results confirm the deductions of our previous reports. (C) 2011 Elsevier B.V. All rights reserved.
\end{abstract}

Keywords: Metamaterials; Bianisotropy; Omega media; Effective parameters

\section{Introduction}

Metamaterials have attracted much attention from the scientific community because of their exotic properties, which usually do not exist in natural materials. For instance, metamaterials with a negative refractive index (NRI) [1-4] can be used to construct a superlens [5-9]. More interestingly, metamaterials can even be used to construct an invisible cloak $[10,11]$. In order to realize such novel properties, metamaterials usually include two types of elements and, therefore, are called composite metamaterials (CMM). For instance, when constructing a metamaterial with NRI, one can use a periodic thin metallic wire medium in order to

\footnotetext{
* Corresponding author. Tel.: +90 312 2901018; fax: +903122901018 .

E-mail address: zhaofengli@bilkent.edu.tr (Z. Li).
}

obtain negative permittivity [12], and use split ring resonators (SRRs) in order to obtain negative permeability [13]. Although SRRs are widely used in metamaterials operating in the microwave region, studies show that there can be problems when SRRs are used at optical frequencies [14]. Bianisotropy usually should be avoided during the design of metamaterials. However, it was proposed recently [15] that it is possible to benefit from the bianisotropic properties of an example metamaterial $-\Omega$ medium (omega medium). An omega medium was first introduced by Saadoun and Engheta [16] and was called a pseudochiral medium in 1992. An omega medium is a composite electromagnetic material with $\Omega$-shaped metallic inclusions that are placed in a host dielectric medium. In the omega medium, there is magnetoelectric coupling due to its intrinsic bianisotropic characteristics. Following the ideas described above $[15,16]$, we studied and reported on a series of 
metamaterials based on $\Omega$-shaped metallic inclusions experimentally and numerically $[17,18]$. The results have clearly shown the differences from the traditional metamaterials made of SRRs. A transmission band was observed for a CMM based on an omega medium, which is below the plasmonic frequency of the closed composite metamaterial (CCMM), in which it has been deduced that this transmission band is not a band of negative refraction, although the effective parameters for the bianisotropic metamaterials were not presented in previous reports.

It has been proposed that metamaterials respond to electromagnetic radiation as continuous materials when the wavelength is much larger than the spacing between the composite components and the size of these respective components. Therefore, it is reasonable to assign values of permittivity $\varepsilon$ and permeability $\mu$ for a metamaterial. Several methods [19-22] have been proposed to retrieve the effective parameters for isotropic metamaterials. Among them, the method based on the scattering parameters (S parameters) of a slab of metamaterial is widely used [23-26]. Compared with isotropic metamaterials, the retrieval of the effective parameters for a bianisotropic metamaterial is more complex due to the existence of the magnetoelectric coupling effect. However, it is proposed that by using the $\mathrm{S}$ parameters of three orthogonal directions [27], it is possible to retrieve all of the effective parameters for a bianisotropic metamaterial. Very recently, our study revealed that it is also possible to retrieve all of the effective parameters for a bianisotropic metamaterial by using the $S$ parameters of only one direction [28]. In the present paper, we will recount some of the brief conclusions of our retrieval method. Then, we will provide the effective parameters including the magnetoelectric coupling coefficient for the bianisotropic omega medium. Our retrieval results validate what was deduced in our previous reports $[17,18]$.

\section{Experimental and numerical results}

Fig. 1(a) and (b) shows two unit cells of the omega medium and the CMM with omega structure inclusions under study. Fig. 1(c) shows the details of the omega structure. The parameters in the figure are $r=1.19 \mathrm{~mm}$, $W=0.45 \mathrm{~mm}$, and $L=1.8 \mathrm{~mm}$. The omega structures are made of copper on a FR4 printed circuit board (PCB). The dielectric constant and the conductance of the FR-4 board are 4.4 and $0.0068 \mathrm{~S} / \mathrm{m}$, respectively. The thickness of the copper and FR4 are $30 \mu \mathrm{m}$ and $1.6 \mathrm{~mm}$, respectively. By arranging these omega
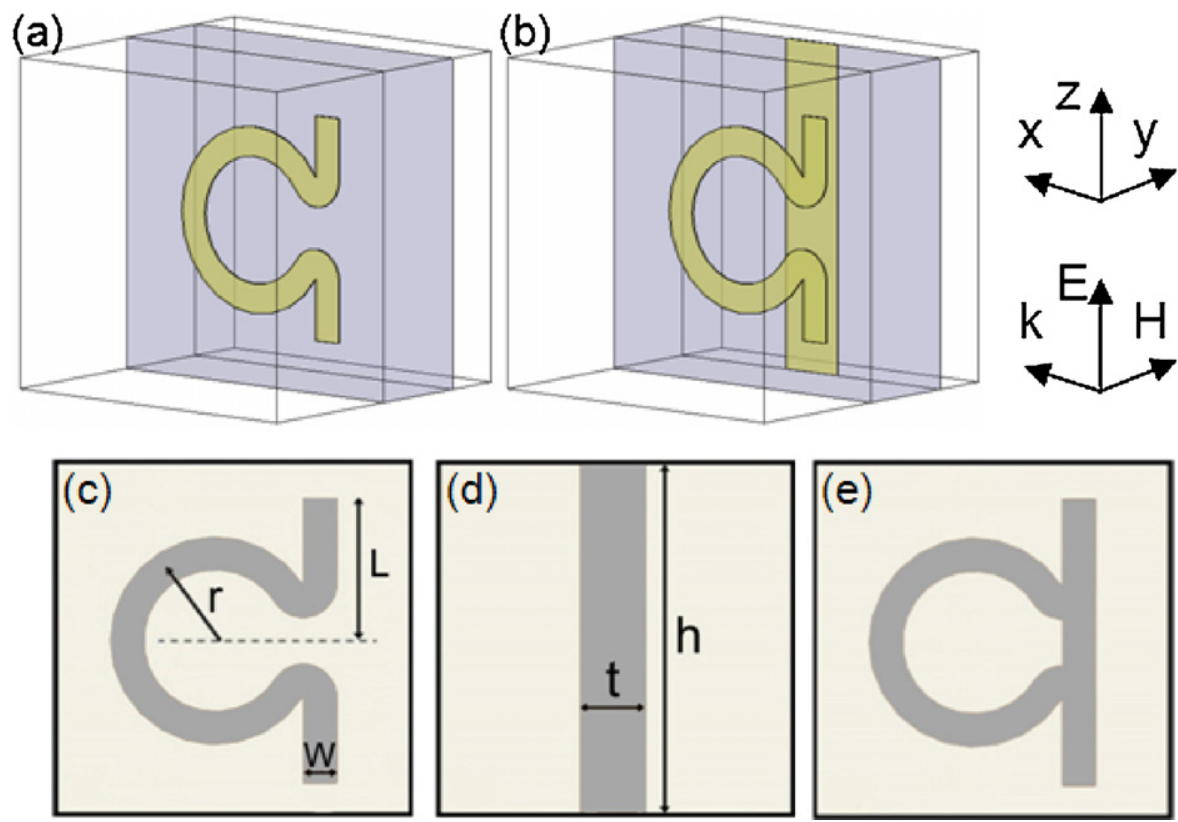

Fig. 1. ( $a$ and b) The schematics of unit cells for the omega medium and the CMM with omega structure inclusions. (c) The omega structure. The dimensions of the omega structure are $r=1.19 \mathrm{~mm}, W=0.45 \mathrm{~mm}$, and $L=1.8 \mathrm{~mm}$, respectively. (d) The continuous wire structure with its dimensions $t=1.44 \mathrm{~mm}$ and $h=5 \mathrm{~mm}$. (e) The closed omega structure, in which its dimensions are equal to the omega structure in (c). The lattice constants for all of the structures are $a_{x}=a_{y}=a_{z}=5 \mathrm{~mm}$. 

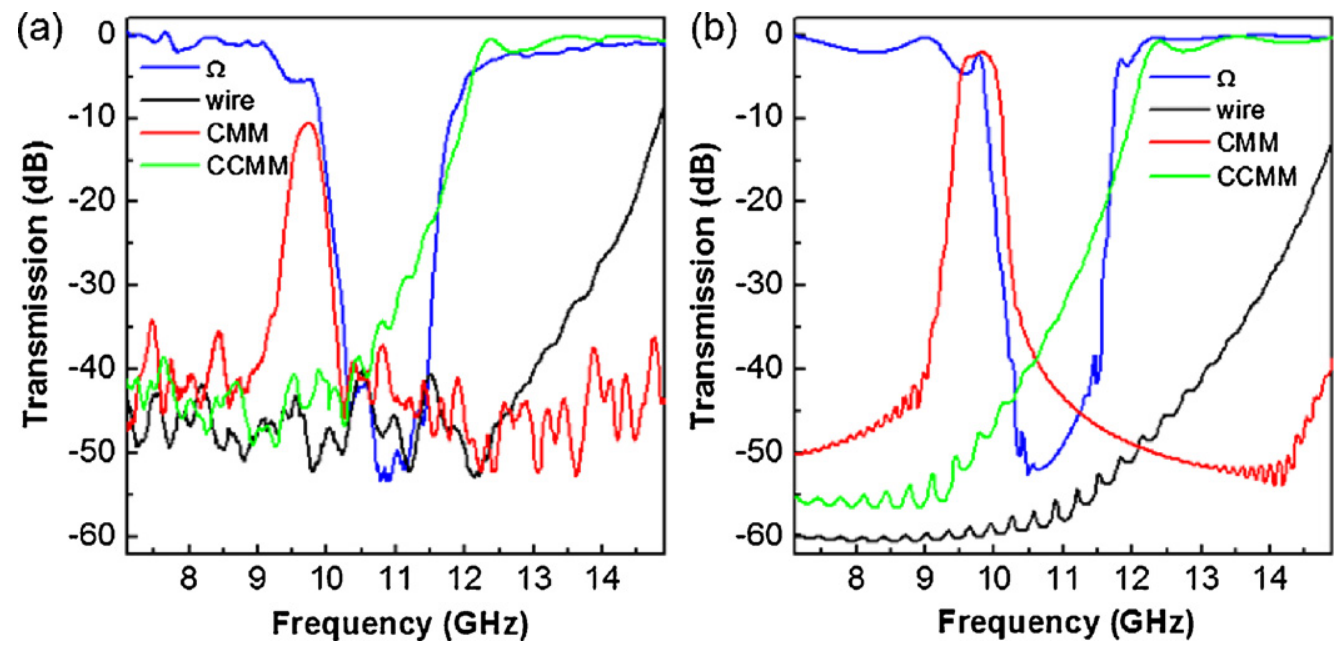

Fig. 2. (a) The transmission spectra of experimental data for the omega medium, periodic wire medium, composite metamaterial, and closed composite metamaterial. (b) The transmission spectra of the simulation results for the same four media.

structures periodically in three orthogonal directions, an omega medium can be obtained. In the experiments, we arrange $\Omega$-resonator units periodically with 5, 40, and 30 unit cells in the $x$-, $y$-, and $z$-directions, respectively. The lattice constants are $a_{x}=a_{y}=a_{z}=5 \mathrm{~mm}$. While in the simulations, we use periodic boundary conditions in the $y$ and $z$ directions. We performed the numerical simulations by using a commercial software package (CST STUDIO microwave) that is based on the finite integration technique. In order to investigate the properties of CMM based on omega structures, a periodic arrangement of continuous thin copper wires were adopted to achieve negative permittivity at microwave frequencies. A unit cell of the continuous wire is shown in Fig. 1(d). The wire is on the opposite side of the PCB. The thickness of the metal is $30 \mu \mathrm{m}$. The width of the thin wire is $t=1.44 \mathrm{~mm}$, and the height is $h=5 \mathrm{~mm}$, which is equal to the periodic constant in the $y$ direction. In our experiments, the lattice constants and number of layers of continuous metallic wires are equal to that of the periodic omega media in the $x$ - and $z$-directions. While in the $z$ direction, the wires are continuous and the total length of the wires is $150 \mathrm{~mm}$.

It was proposed [29] that by using closed (or shorted) resonators combined with periodic metallic wires one can obtain the rectified plasmonic frequency. If a transmission band of the corresponding CMM is below the rectified plasmonic frequency, this transmission band can be thought of as a band of negative refraction. Accordingly, the closed $\Omega$ structure shown in Fig. 1(e) is adopted in our study as a reference. During the experiments, transmission measurements were performed in free space by using an HP 8510-C network analyzer. Microwave horn antennas were used as transmitters and receivers, in which the transmission through the samples was measured.

Fig. 2(a) and (b) shows the results of the experiment and simulation, respectively. Our simulation results are in good agreement with that of the experiment. From Fig. 2, it can be clearly seen that there is a transmission band for the CMM medium, which is below the plasmonic frequency of the corresponding CCMM medium. According to Aydin et al. [29], this transmission band is much likely a band of negative refraction. However, unlike the situations in composite metamaterials based on SRRs, this transmission band of CMM does not coincide with the transmission gap of the corresponding omega medium. Instead, this transmission band shifts to the lower frequency end. The above phenomenon is quite similar to the transmission behavior of a $\pi$-structure [30], which is also deduced to not be a left-handed transmission. Therefore, the transmission band of the CMM based on the omega structure is considered to be right-handed in our previously conducted reports $[17,18]$. In the next section, we will retrieve the effective parameters for omega-based metamaterials and see whether our previous conclusion was correct or not.

\section{Retrieval results}

For the omega medium shown in Fig. 1(a), by assuming that the medium is reciprocal $[31,32]$ and that the harmonic time dependence is $e^{-i \omega t}$, where $\omega$ is an 
angular frequency, we can write the constitutive relationships as follows:

$\vec{D}=\overline{\bar{\varepsilon}} \cdot \vec{E}+\overline{\bar{\xi}} \cdot \vec{H}$

$\vec{B}=\overline{\bar{\mu}} \cdot \vec{H}+\overline{\bar{\zeta}} \cdot \vec{E}$

where

$\overline{\bar{\varepsilon}}=\varepsilon_{0}\left(\begin{array}{ccc}\varepsilon_{x} & 0 & 0 \\ 0 & \varepsilon_{y} & 0 \\ 0 & 0 & \varepsilon_{z}\end{array}\right), \quad \overline{\bar{\mu}}=\mu_{0}\left(\begin{array}{ccc}\mu_{x} & 0 & 0 \\ 0 & \mu_{y} & 0 \\ 0 & 0 & \mu_{z}\end{array}\right)$,

$\overline{\bar{\xi}}=\frac{1}{c}\left(\begin{array}{ccc}0 & 0 & 0 \\ 0 & 0 & 0 \\ 0 & -i \xi_{0} & 0\end{array}\right), \quad \bar{\zeta}=\frac{1}{c}\left(\begin{array}{ccc}0 & 0 & 0 \\ 0 & 0 & i \xi_{0} \\ 0 & 0 & 0\end{array}\right)$

where $\varepsilon_{0}$ and $\mu_{0}$ are the permittivity and permeability of the vacuum, respectively, in which $c$ is the speed of light in vacuum. The seven unknowns, $\varepsilon_{x}, \varepsilon_{y}, \varepsilon_{z}, \mu_{x}, \mu_{y}, \mu_{z}$, and $\xi_{0}$ are quantities without a dimension. When a plane wave that is polarized in the $z$ direction is incident in the $x$ direction, three parameters $\left(\varepsilon_{z}, \mu_{y}\right.$, and $\left.\xi_{0}\right)$ will be active, while the other four parameters $\left(\varepsilon_{x}, \varepsilon_{y}, \mu_{x}\right.$, and $\left.\mu_{z}\right)$ will not be involved in the bianisotropic process and, therefore, are out of the scope of the present study. According to the formulae in Ref. [31], one can easily obtain the expressions for the effective constitutive parameters based on $\varepsilon_{z}$, $\mu_{y}$, and $\xi_{0}$. However, there is one main thing that one should note. Compared to an isotropic material, the most interesting and important feature of a bianisotropic material is that the characteristic impedances have different values for the waves propagating in the two opposite directions of the $x$ axis. For an electromagnetic (EM) wave traveling in the $\pm x$ direction, the impedances will be

$z^{+}=\frac{\mu_{y}}{n+i \xi_{0}}, \quad z^{-}=\frac{\mu_{y}}{n-i \xi_{0}}$

respectively. Where $n$ is the effective refractive index, which has the same value for the EM wave traveling in two opposite directions of the $x$ axis:

$n= \pm \sqrt{\varepsilon_{z} \mu_{y}-\xi_{0}^{2}}$

Our recent study [28] has found a simpler method to retrieve the effective parameters for a bianisotropic metamaterial compared to the method proposed in Ref. [27]. According to our study, in order to retrieve the effective parameters of a bianisotropic medium, one only needs to know the $\mathrm{S}$ parameters in the two directions (say $\pm x$ directions for the present case) with the incident waves polarized in the $z$ direction. Consequently, one obtains the analytical expression for the refractive index $n$, which is

$\cos \left(n k_{0} l\right)=\frac{1-S_{11} S_{22}+S_{21}^{2}}{2 S_{21}}$

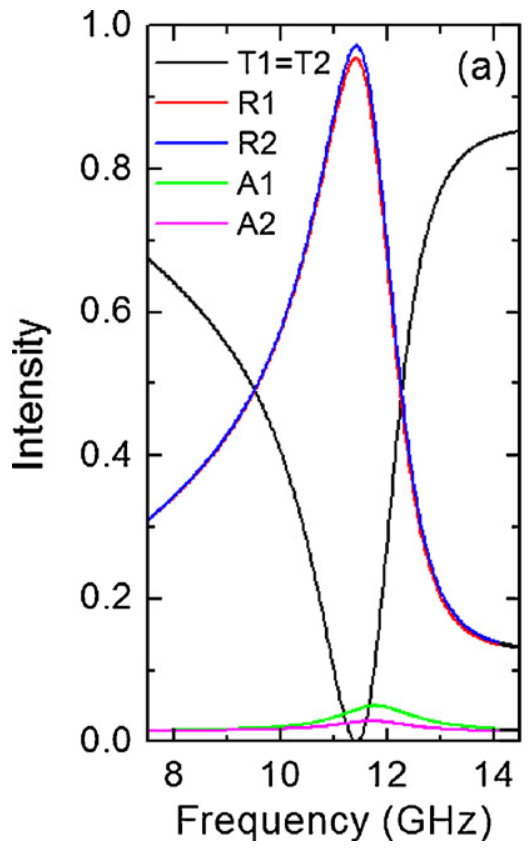

Fig. 3. The intensity spectra of transmission, reflection, and absorption for the omega (a) and the CMM (b) media. R1 and R2 (A1 and A2, T1 and $\mathrm{T} 2$ ) are the reflection (absorption, transmission) spectra when the waves are incident in the $+x$ and $-x$ directions, respectively. 
where $k_{0}$ is the wave number of the EM wave in vacuum, and $l$ is the thickness of the bianisotropic medium. When solving $n$ from Eq. (5), one must determine one branch from many branches of solutions. Fortunately, there have been several reports $[22,26]$ dealing with this problem. Therefore, we will not detail it here. For a passive medium, the solved $n$ must follow the following condition:

$n^{\prime \prime} \geq 0$

where $(\cdot)^{\prime \prime}$ denotes the imaginary part operator. After $n$ is solved, other constitutive parameters can be obtained by the following relations:

$$
\begin{aligned}
& \xi_{0}=\left(\frac{n}{-2 \sin \left(n k_{0} l\right)}\right)\left(\frac{S_{11}-S_{22}}{S_{21}}\right) \\
& \mu_{y}=\left(\frac{i n}{\sin \left(n k_{0} l\right)}\right)\left(\frac{2+S_{11}+S_{22}}{2 S_{21}}-\cos \left(n k_{0} l\right)\right)
\end{aligned}
$$

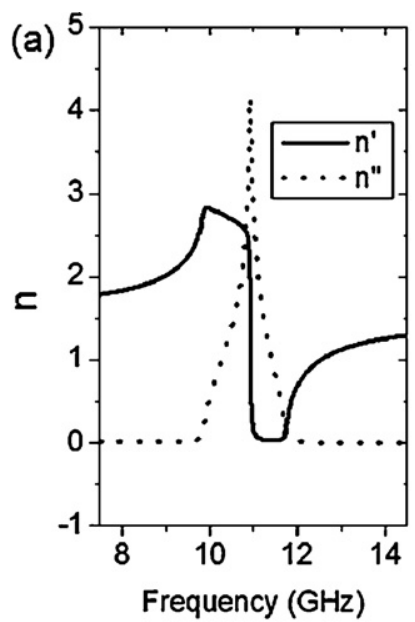

$\varepsilon_{z}=\frac{n^{2}+\xi_{0}^{2}}{\mu_{y}}$

After obtaining the above three parameters $\left(\varepsilon_{z}, \mu_{y}\right.$, and $\left.\xi_{0}\right)$, the impedances $\left(z^{+}\right.$and $\left.z^{-}\right)$can be solved from Eq. (3). Again, for a passive medium, the following conditions should be satisfied,

$z^{+\prime} \geq 0, \quad z^{-\prime} \geq 0$,

where $(\cdot)^{\prime}$ denotes the real part operator. So far, all of the constitutive parameters that are related to bianisotropy were retrieved. In the following subsections, we will provide the retrieval result for the omega medium as well as the CMM based on it. In the retrieval procedure, we employed a single layer of omega medium (or $\mathrm{CMM}$ ) along the $x$-axis. Hence, the simulation setup coincides with a slab of omega medium (or CMM) that consists of single period layer. The effective parameters were then derived from the transmission and reflection

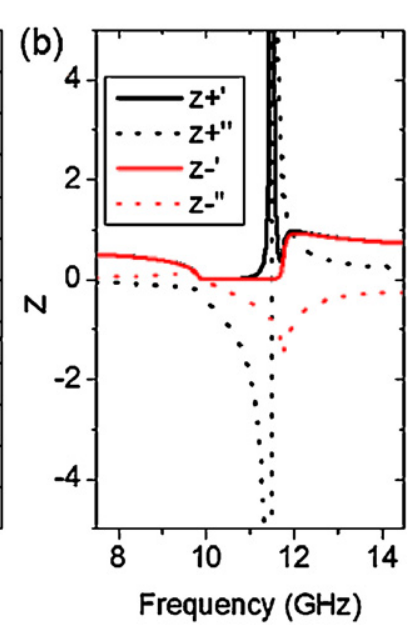

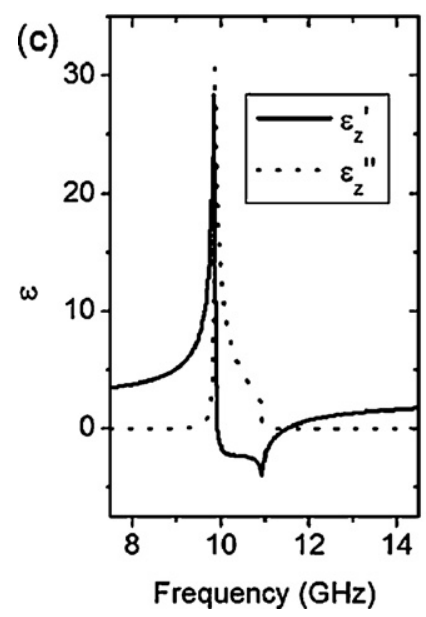
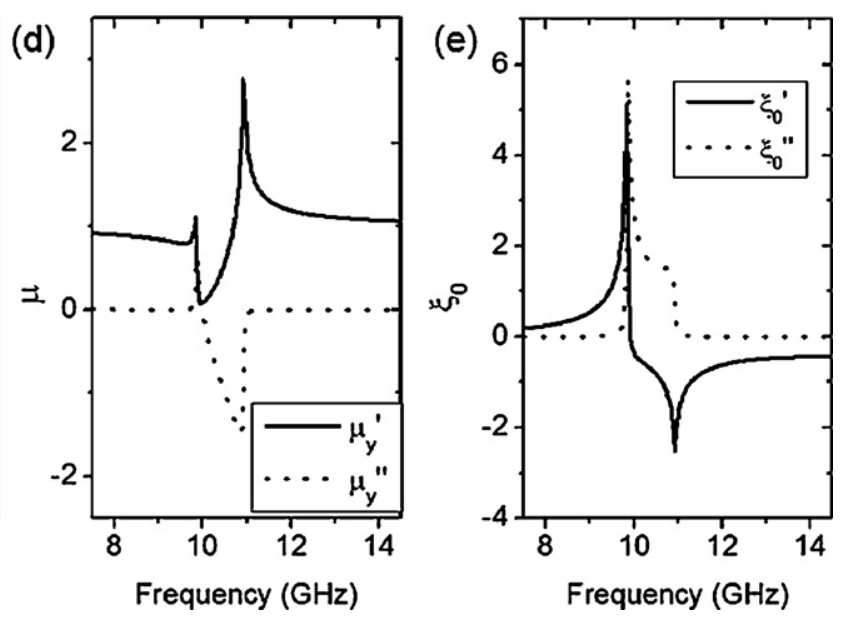

Fig. 4. The retrieval results of the effective parameter for the bianisotropic omega medium. (a) Effective refractive index $n$. (b) Impedances $z^{+}$and $z^{-}$. (c) Permittivity $\varepsilon$. (d) permeability $\mu$. (e) Magnetoelectric coupling efficient $\xi_{0}$. 
coefficients of this single layer of omega medium (or CMM).

\subsection{S parameters and absorption spectra of the omega and the CMM media}

Using the structural dimensions for the omega and the CMM media that was presented in the previous sections, we calculated the $\mathrm{S}$ parameters of one period of the two media for the two opposite directions along the $x$-axis, and the intensity spectra are shown in Fig. 3. In Fig. 3, we also show the absorption spectra of the two media. It is seen that the reflection and absorption spectra are different when the EM waves are incident in the $+x$ and $-x$ directions. This phenomenon happens due to the lossy substrate and the bianisotropy of the two media, as can be well explained in Eqs. (5)-(8) of the Ref. [28].
Moreover, it is also seen from Fig. 3(a) that the peaks of the absorption spectra of the omega medium are shifted to higher frequencies compared to that of the reflection spectra. This deference of the peak frequencies between the scattering and absorption spectra has been found for varied scattering particles [33]. This phenomenon will be further studied in our future works.

\subsection{Retrieval results of the omega medium}

Fig. 4 shows the retrieval results for the omega medium based on the calculated S parameters. Fig. 4(a) and (b) illustrates the results of the effective refractive index $n$ and impedances $z^{+}$and $z^{-}$, respectively. In a wide frequency range, $z^{+}$and $z^{-}$definitely have different values. Fig. 4(c) and (d) shows the retrieval results of permittivity and permeability, respectively. The omega medium shows strong features of electric
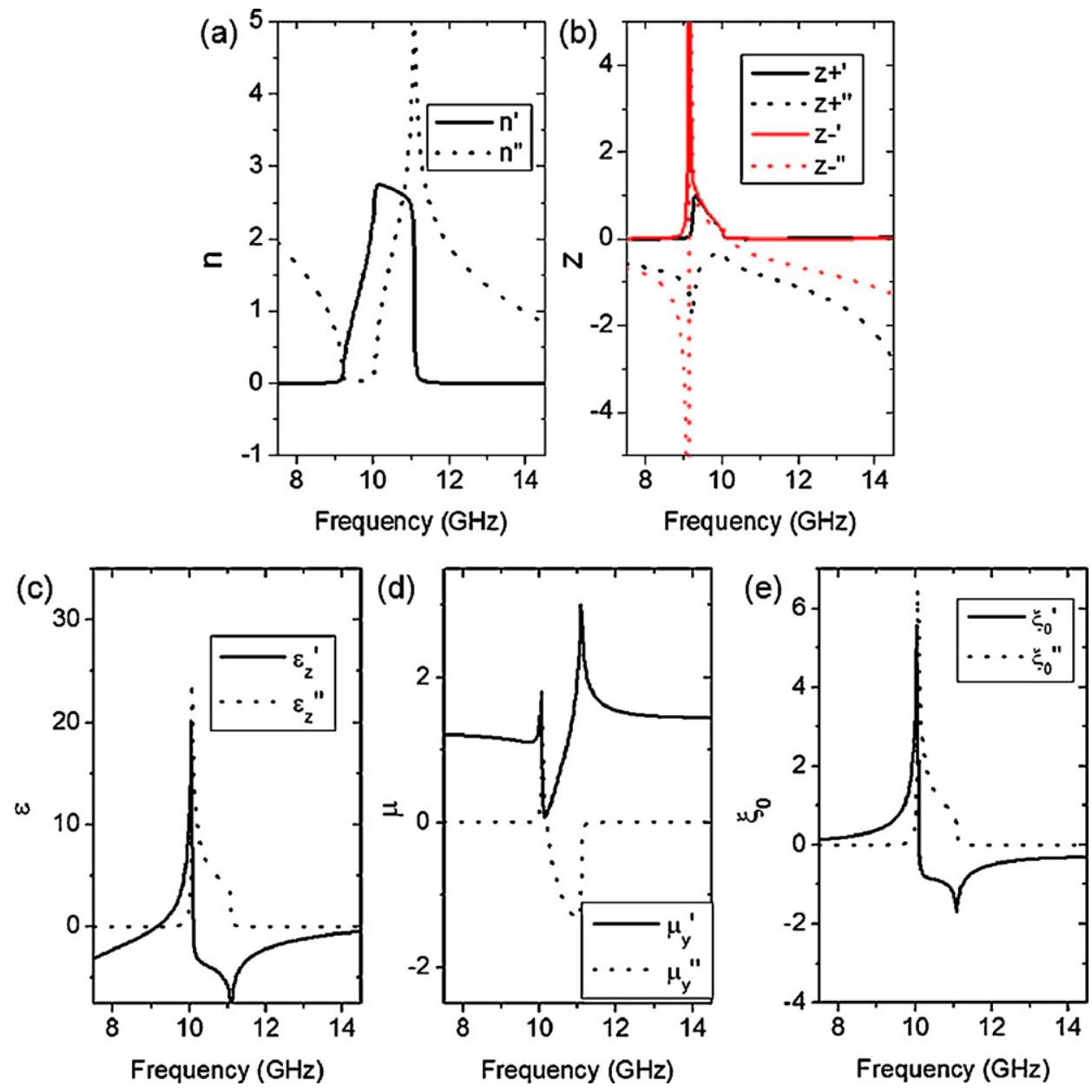

Fig. 5. The retrieval results of the effective parameter for the bianisotropic CMM based on the $\Omega$ medium. (a) Effective refractive index $n$. (b) Impedances $z^{+}$and $z^{-}$. (c) Permittivity $\varepsilon$. (d) Permeability $\mu$. (e) Magnetoelectric coupling efficient $\xi_{0}$. 
resonance rather than magnetic resonance, which is quite different from the results of SRR media [22]. Moreover, one did not see any frequency range with negative permeability. Fig. 4(e) shows the retrieval result for the magnetoelectric coupling coefficient. It can be seen clearly that near the resonant frequency, there obviously exists a strong magnetoelectric coupling in the omega medium. The existence of the strong magnetoelectric coupling results in the different impedances of $z^{+}$and $z^{-}$as shown in Fig. 4(b).

\subsection{Retrieval results of the CMM medium}

Fig. 5 shows the retrieval results for the CMM medium, which is a combination of the omega structures and infinite wire structures. Fig. 5(a) shows the retrieved effective refractive index $n$ for the CMM medium. One sees that there is a frequency range (9.2$10.1 \mathrm{GHz}$ ) with positive $n^{\prime}$ but very small $\mathrm{n}^{\prime \prime}$, which exactly corresponds to the transmission band of the CMM shown in Fig. 2. Fig. 5(b) shows the results for the impedances $z^{+}$and $z^{-}$, in which again the two impedances have different values. Fig. 5(c) shows the results of the permittivity. By comparing the data shown in Fig. 4(c), one sees that even though the CMM medium includes periodic thin wire structures that provide negative permittivity, there still exists a frequency range $(9.2-10.1 \mathrm{GHz})$ with positive permittivity. This frequency range also corresponds to the transmission band of the CMM medium as shown in Fig. 2. Fig. 5(d) and (e) illustrates the retrieved results for the permeability and magnetoelectric coupling coefficient, respectively. These two sets of data are quite similar to that of the omega medium as shown in Fig. 4(d) and (e), which means that the addition of the thin wire structure into the omega medium only has little influence on the two parameters of $\mu_{y}$ and $\xi_{0}$.

According to the retrieval results for the omega and CMM media, it is concluded that the transmission band of the CMM medium below the plasmonic frequency of CCMM is a band with positive refractive indices. Our retrieval results confirm what we deduced in our previous reports [17,18]. Furthermore, our investigations on the omega medium illustrate that for a bianisotropic metamaterial it is insufficient to decide whether a transmission band of a CMM is of a positive or negative refractive index by comparing it to the transmission spectrum of a corresponding CCMM. Although we did not obtain a metamaterial with NRI based on an omega medium here, our retrieval method and procedure revealed the underlying physics of the omega medium and its corresponding CMM medium.
By using the retrieval procedure as a tool, it will be easier to tailor the properties of omega-related media and bianisotropic metamaterials in future studies.

\section{Conclusions}

In the present paper, we retrieved the effective parameters for an omega medium and its corresponding CMM. Since omega-related media are intrinsically bianisotropic, we retrieved not only the parameters of permittivity and permeability but also the magnetoelectric coupling coefficient that measures the intensity of the bianisotropy. Furthermore, we also obtained the effective parameters of the refractive index and impedances. Based on our retrieval results, it was found that the transmission band of the CMM below the plasmonic frequency of its corresponding CCMM is a band with positive refractive indices. The retrieval results confirm what was deduced in our previous reports.

\section{Acknowledgments}

This work is supported by the European Union under the projects DPT-HAMIT, EU-PHOME, EU-N4E, NATO-SET-181 and TUBITAK under Project Nos. 107A004, 107A012, 109E301. One of the authors (E.O.) also acknowledges partial support from the Turkish Academy of Sciences.

\section{References}

[1] R.A. Shelby, D.R. Smith, S. Schultz, Science 292 (2001) 77-79.

[2] A.A. Houck, J.B. Brock, I.L. Chuang, Phys. Rev. Lett. 90 (2003) 137401.

[3] K. Guven, C.M. Soukoulis, Appl. Phys. Lett. 86 (2005) 124102.

[4] K. Aydin, E. Ozbay, J. Opt. Soc. Am. B 23 (2006) 415-418.

[5] N. Fang, H. Lee, C. Sun, X. Zhang, Science 308 (2005) 534-537.

[6] D.O.S. Melville, R.J. Blaikie, Opt. Express 13 (2005) 2127-2134.

[7] K. Aydin, I. Bulu, E. Ozbay, Opt. Express 13 (2005) 8753-8759.

[8] K. Aydin, I. Bulu, E. Ozbay, New J. Phys. 8 (2006) 221.

[9] Z. Liu, S. Durant, H. Lee, Y. Pikus, N. Fang, Y. Xiong, C. Sun, X. Zhang, Nano Lett. 7 (2007) 403-408.

[10] J.B. Pendry, D. Schurig, D.R. Smith, Science 312 (2006) 17801782.

[11] D. Schurig, J.J. Mock, B.J. Justice, S.A. Cummer, J.B. Pendry, A.F. Starr, D.R. Smith, Science 314 (2006) 977-980.

[12] J.B. Pendry, A.J. Holden, W.J. Stewart, I. Youngs, J. Phys. Condens. Matter. 10 (1998) 4785-4809.

[13] J.B. Pendry, A.J. Holden, D.J. Robbins, W.J. Stewart, I.E.E.E. Trans, Microw. Theory Technol. 47 (1999) 2057.

[14] A. Alu, A. Salandrino, N. Engheta, Opt. Express 14 (2006) $1557-1567$

[15] S.A. Tretyakov, C.R. Simovski, M. Hudlicka, Phys. Rev. B 75 (2007) 153104. 
[16] M.M.I. Saadoun, N. Engheta, Microwave Opt. Technol. Lett. 5 (1992) 184-188.

[17] K. Aydin, Z. Li, M. Hudlicka, S.A. Tretyakov, E. Ozbay, New J. Phys. 9 (2007) 326.

[18] K. Aydin, Z. Li, S. Bilge, E. Ozbay, Photon. Nanostruct. 6 (2008) $116-121$

[19] J.M. Lerat, N. Mallejac, O. Acher, J. Appl. Phys. 100 (2006) 084908.

[20] B. Popa, S.A. Cummer, Phys. Rev. B 72 (2005) 165102.

[21] R. Marques, F. Medina, R. Rafii-El-Idrissi, Phys. Rev. B 65 (2002) 144440.

[22] D.R. Smith, S. Schultz, P. Markos, C.M. Soukoulis, Phys. Rev. B 65 (2002) 195104.

[23] T. Koschny, P. Markos, D.R. Smith, C.M. Soukoulis, Phys. Rev. E 68 (2003), 065602(R).

[24] D.R. Smith, D.C. Vier, T. Koschny, C.M. Soukoulis, Phys. Rev. E 71 (2005) 036617.
[25] T. Koschny, P. Markos, E.N. Economou, D.R. Smith, D.C. Vier, C.M. Soukoulis, Phys. Rev. B 71 (2005) 245105.

[26] X. Chen, T.M. Grzegorczyk, B.-I. Wu, J. Pacheco, J.A. Kong, Phys. Rev. E 70 (2004) 016608

[27] X. Chen, B.-I. Wu, J.A. Kong, T.M. Grzegorczyk, Phys. Rev. E 71 (2005) 046610.

[28] Z. Li, K. Aydin, E. Ozbay, Phys. Rev. E 79 (2009) 026610.

[29] E. Aydin, K. Guven, M. Kafesaki, L. Zhang, C.M. Soukoulis, E. Ozbay, Opt. Lett. 29 (2005) 2623-2625.

[30] Z.-G. Dong, S.-Y. Lei, M.-X. Xu, H. Liu, T. Li, F.-M. Wang, S.N. Zhu, Phys. Rev. B 75 (2007) 075117.

[31] J.A. Kong, Electromagnetic Wave Theory, EMW, Cambridge, MA, 2008

[32] S.A. Tretyakov, A.A. Sochava, Electron. Lett. 29 (1993) 10481049.

[33] J. Zhu, J. Li, J. Zhao, Appl. Phys. Lett. 99 (2011) 101901. 